\title{
Objeto de aprendizagem reutilizável em 3D para a identificação de Craniosynostosis: um estudo de caso
}

\author{
Bianca Carneiro Ribeiro - UFRPE - bianca.ead@gmail.com \\ Jorge da Silva Correia-Neto - UFRPE - jorgecorreianeto@gmail.com \\ Ronnie Edson de Souza Santos - CIn - UFPE, ress@cin.ufpe.br \\ Clayton Vanut Cordeiro de Magalhães - CIn - UFPE, cvcm@cin.ufpe.br \\ Guilherme Vilar - UFRPE - guilherme_vilar@yahoo.com.br
}

\begin{abstract}
In the context that involves medical education, learning objects have been used as a practical, economic and robust technology. This study aims to develop and evaluate learning objects using tridimensional images in detections of craniofacial dysmorphology. The learning object was developed by following steps: modeling, texturing, animations and rendering. The next stage was an evaluation made by a group of pediatricians using learning software. The results present a set of high quality tridimensional animations that show variations of a head with dysmorphology. The results also perform a set of discussions about the importance that 3D learning objects may have in medical education.
\end{abstract}

Resumo. No contexto que envolve a educação médica continuada, os objetos de aprendizagem tem se mostrado uma tecnologia bastante robusta, prática e econômica. Neste sentido, esta pesquisa tem o objetivo de desenvolver e avaliar objetos de aprendizagem utilizando imagens tridimensionais na detecção de dismorfologias craniofaciais. O objeto de aprendizagem foi construído seguindo as etapas de modelagem, texturização, animação e renderização, com posterior avaliação por um grupo de pediatras através de um ambiente virtual de aprendizagem. Os resultados reúnem um conjunto de animações tridimensionais de alta qualidade demonstrando a variação de uma cabeça com dismorfologias e um conjunto de discussões sobre a importância que os objetos de aprendizagem em 3D podem ter na educação médica.

\section{Introdução}

De acordo com Silva (2001), a realidade virtual surgiu como uma opção de lazer, onde a possibilidade do usuário fazer parte de um mundo diferente do seu tornou-se atrativa. É através da realidade virtual e da computação gráfica que tem se tornado possível a criação de mundos virtuais e, diante disso, esta abordagem se tornou uma ferramenta poderosa para as mais diversas áreas. A realidade virtual é, antes de tudo, uma 'interface avançada de usuário’ para acessar aplicações executadas no computador, tendo como características a visualização de, e movimentação em, ambientes tridimensionais em tempo real e a interação com elementos desse ambiente (TORI; KIRNER, 2006).

Desde o início da computação gráfica, a medicina vem sendo uma das muitas áreas beneficiadas com aplicações, como já apontavam El-Khalili e Brodlie (1998). A medicina é uma área de pesquisa extremamente desafiante e de fundamental importância na existência humana. A variedade e complexidade inerente de problemas ainda não solucionados fazem disso uma força direcionada para muitas engenharias e ciências naturais. Ferramentas e métodos gráficos individuais tornaram-se, assim, cada vez mais 
insubstituíveis na medicina moderna, onde os sistemas de imagem médica são apenas um proeminente exemplo (GROSS, 1998).

Na educação médica continuada, a tecnologia também se apresenta como promissora opção para suprir necessidades e dar suporte a possibilidades como maior intercâmbio de informações entre profissionais da área médica potencializando o aprendizado e redução de custo, entre outras (VILAR; VILAR, 2004; BURITY; CARDOZO, 2014). Além disso, as pesquisas realizadas na área da Informática na Educação certamente vêm contribuindo para o surgimento de novas ferramentas que possibilitam uma participação mais ativa do estudante de medicina (SEBASTIANI; BEZ; FLORES, 2009).

De acordo com Dev (1999), um dos principais objetivos da educação médica é o de ensinar a detecção e resolução de problemas (diagnóstico) com base em amplos conhecimentos, experiência e análise de cada doente. Neste contexto, os objetos de aprendizagem têm se mostrado uma tecnologia bastante robusta, prática e econômica para esta área, como discutido anteriormente por Ferreira (2004) e por Santa-Rosa e Struchiner (2011).

Um objeto de aprendizagem pode ser definido como qualquer entidade, digital ou não-digital, que pode ser usada, reutilizada ou referenciada durante o processo de aprendizagem apoiado por tecnologias (IEEE, 2000). Um objeto de aprendizagem pode conter desde elementos simples como texto ou vídeo até a aplicação de hipertexto, animações com áudio e recursos mais complexos de programação (WILEY, 2000; GLUZ; VICARI, 2010). A principal característica do objeto de aprendizagem é sua reusabilidade, que é posta em prática através de repositórios, que armazenam os objetos, permitindo serem localizados a partir da busca por temas, por nível de dificuldade, por autor ou por relação com outros objetos (SANTOS, 2007).

Além disso, a pesquisa sobre objetos de aprendizagem vem crescendo significativamente nos últimos anos no Brasil, tanto com objetos de instrução (dedicados a auxiliar no processo de aprendizagem), como com os de colaboração (presentes nos ambientes de aprendizagem colaborativa), os de prática (autoaprendizagem) e os de avaliação (BESERRA et al., 2014).

Por outro lado, sabe-se que, na área da medicina, a genética é um tema que aborda assuntos complexos, tais como as dismorfologias. A dismorfologia é um ramo da genética médica que estuda malformações congênitas humanas que literalmente significa "o estudo da forma anormal” enfatizando anormalidades físicas do desenvolvimento (MEDICINENET.COM, 2008). Entre essas anormalidades existem as dismorfologias do crânio e da face, que constituem o grupo mais diverso e complexo. Muitas dessas dismorfologias podem ser indícios significativos na identificação inicial das condições genéticas de um paciente (HAMMOND, 2007), pois essas malformações podem estar associadas a uma síndrome. Detectar corretamente e precocemente características dismorfológicas no paciente leva a um possível diagnóstico de síndrome, alterando significativamente o tratamento da mesma. Para isso é necessário que o médico possua certo conhecimento sobre as malformações congênitas existentes. Durante o processo de aprendizado destes conteúdos, as imagens de visualização constituem um elemento essencial da aprendizagem de conteúdos. 
Considerando que a tecnologia é atualmente um elemento de grande importância no processo de aprendizagem, pode-se tirar vantagem de uma grande quantidade de material visual para tornar o aprendizado mais eficiente e mais envolvente. Dessa forma, surge a necessidade de aprimorar o estudo das dismorfologias através da criação e avaliação de objetos de aprendizagem interativos, contendo informações escritas de modo claro, objetivo e simples, com imagens que se aproximassem o máximo possível da realidade, facilitando a percepção e deteç̧ão das malformações congênitas, relacionando termos e conceitos através de imagens tridimensionais representativas.

A partir desta introdução, este artigo segue organizado em três seções. A segunda seção apresenta as etapas metodológicas da pesquisa. Logo após, na terceira seção, os resultados do estudo são apresentados e discutidos e, por fim, a quarta seção apresenta as conclusões.

\section{Procedimentos metodológicos}

A construção deste objeto de aprendizagem seguiu a execução de três etapas principais: modelagem, texturização, animação e renderização.

\subsection{Modelagem}

A modelagem constituiu a criação e movimentação de pontos no espaço, através das três coordenadas em 3D (x,y, z), constituindo as faces utilizadas no objeto de aprendizagem. Mesmo com as dificuldades de se desenhar formas mais complexas e, especialmente, formas em movimento (CANI, 2014), a literatura prevê a execução de diversos tipos de métodos para a modelagem de objetos que possuem características específicas capazes de facilitar o processo de desenvolvimento de formas geométricas.

Nesta pesquisa, a modelagem de forma livre (SILVEIRA NETO; KREPSKY, 2005) foi o método utilizado na criação das imagens tridimensionais representativas de um ser humano, mais especificamente da cabeça em 3D. Este método trata de um processo em que o desenvolvedor modela uma forma primitiva (figura 1A) através da manipulação de seus vértices, faces e polígonos (figura 1B). A forma primitiva utilizada foi um Box e tentou-se manter a maior parte das faces quadriculares, conforme pode ser observado na figura 2 .
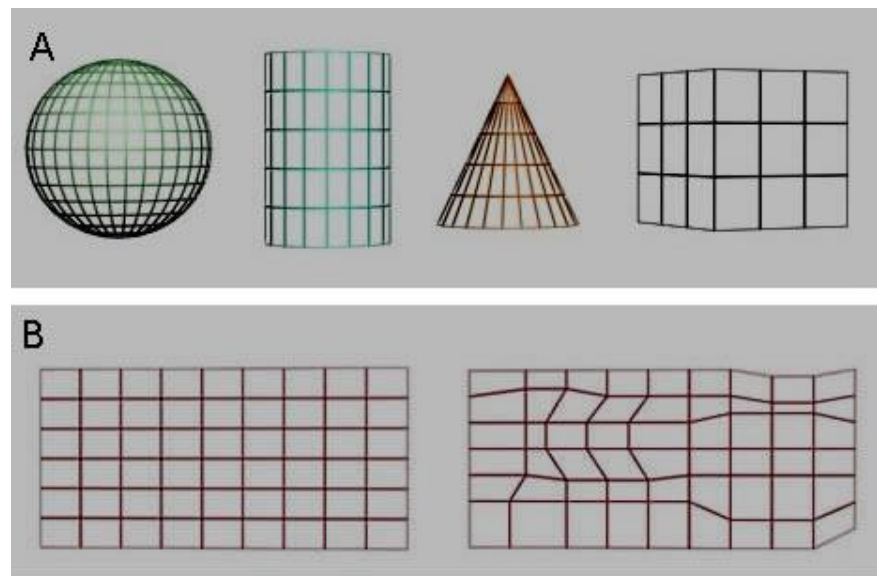

Figura 1. (A) Formas primitivas geométricas. (B) Modelagem de forma livre. 


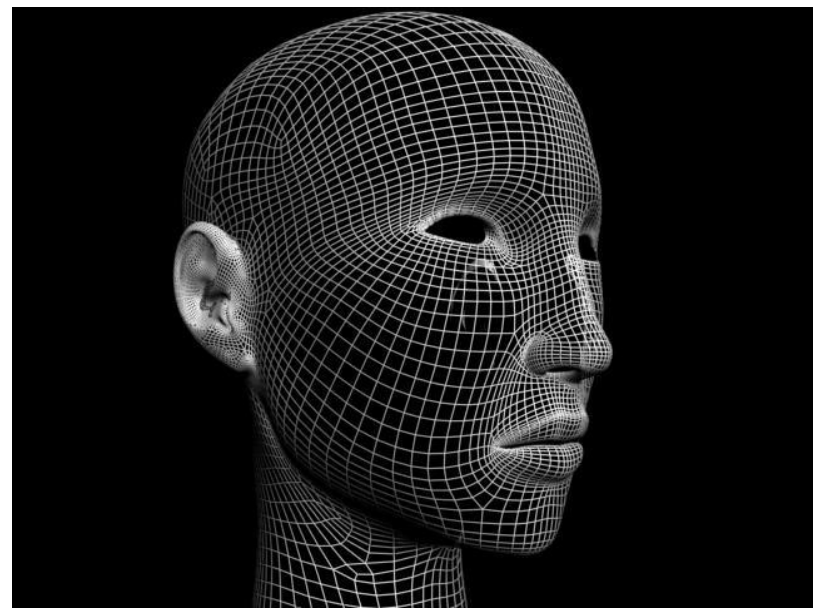

Figura 2. Rosto com a maior parte das faces quadriculares.

Para a criação das deformações da cabeça, foi necessário modelar primeiro uma cabeça considerada perfeita utilizando o programa 3D Studio (figuras 3A e 3C) e a partir deste ponto, criar as deformações do crânio e da face. As deformações do crânio e mandíbula foram criadas utilizando o software ZBrush (figuras 3B e 3D).

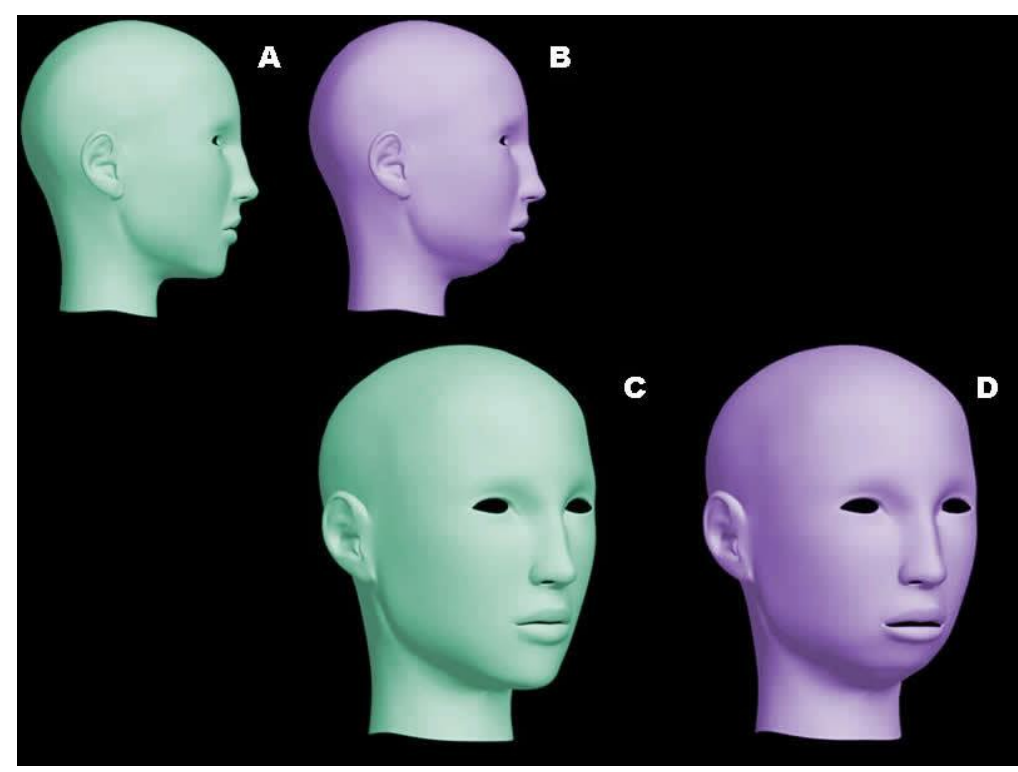

Figura 3. (A) Vista lateral de um rosto considerado perfeito. (B) Vista lateral de um rosto com deformação na mandíbula. (C) Vista em perspectiva do mesmo rosto considerado perfeito. (D) Vista em perspectiva de um rosto com a mesma deformação na mandíbula.

\subsection{Texturização}

A texturização pode ser definida como um tipo de imagem bidimensional ou um material aplicado em um determinado objeto 3D criado. Agregar essa textura aos tecidos modelados visa atribuir realismo ao resultado final e favorecer a percepção de imersão na utilização do simulador, mas esse é um desafio constante para a computação gráfica, 
devido à complexidade das técnicas envolvidas, especialmente na construção de figuras que representem as superfícies do corpo humano (TORI et al., 2014).

A aplicação de texturas respeita o formato do objeto e tem como objetivo proporcionar ao usuário a sensação de estar mais próximo à realidade. Sendo assim, a textura do rosto foi criada utilizando uma ferramenta de edição de imagens e, para tal, utilizaram-se fotos como referência, tendo como base a imagem do mapeamento realizado no 3D Studio, como pode ser observado na figura 4.

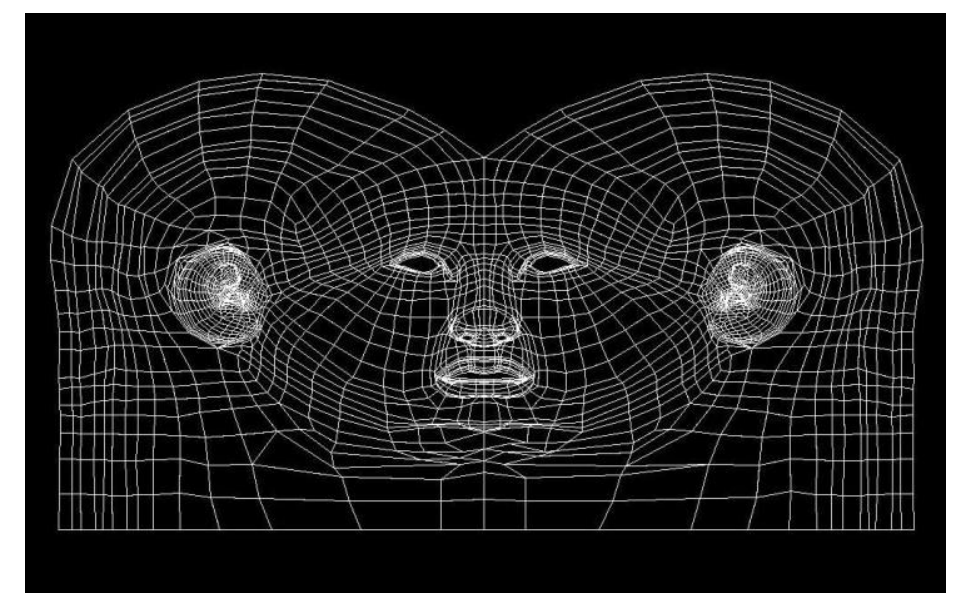

Figura 4. Imagem da malha usada como referência para a criação da textura.

\subsection{Animação e renderização}

A técnica de animação utilizada neste trabalho foi a de interpolação entre quadros chaves (SILVEIRA NETO; KREPSKY, 2005), que permite ao usuário definir apenas os quadros principais da cena, pois o software gera automaticamente os quadros intermediários. Após a criação das imagens tridimensionais da cabeça, tendo-se modelado as deformações, aplicado texturas, definido posições de câmeras e desenvolvidas as devidas animações, segue-se a etapa de renderizar as imagens. Para tal foi necessário renderizar, para cada dismorfologia, imagens em vários ângulos com extensão jpg.

$\mathrm{Na}$ etapa final, as imagens foram importadas para um software de manipulação gráfica vetorial e criação de animações, áudio e vídeo, permitindo a transformação das imagens para um formato em que o usuário pode estabelecer maior grau de interatividade com o objeto. As setas do teclado foram o dispositivo de entrada escolhido para conectar o usuário com o sistema.

\subsection{Avaliação do objeto proposto}

Os objetos de aprendizagem foram disponibilizados em um ambiente virtual para que, posteriormente, fosse realizado um processo de avaliação do resultado final com pediatras cadastrados. A ferramenta escolhida para esta etapa do processo foi o ambiente Moodle ${ }^{i i}$, por ter uma estrutura baseada em módulos, interface intuitiva e facilidade na disponibilização de materiais e inclusão de atividades. 
Os pediatras foram submetidos a uma análise quali-quantitativa efetuada antes e depois da utilização e visualização dos objetos de aprendizagem. Esta análise foi dividida em três módulos: craniossinostose, micrognatia e macrognatia, contendo questões de auto-percepção utilizando uma escala adaptada de Lima Júnior, Knopfholz e Menini (2002). Trata-se de uma abordagem que utiliza um segmento de reta contendo 600 pixels de comprimento sem indicação de valores, com uma pequena barra que se move para a esquerda e direita (figura 5A). O profissional de saúde deverá clicar na posição que represente o seu grau de conhecimento sobre as dismorfologias. Ao clicar, será impresso na tela um valor em pixels (figura 5B), que o usuário deverá anotar e enviar para o ambiente virtual.

A

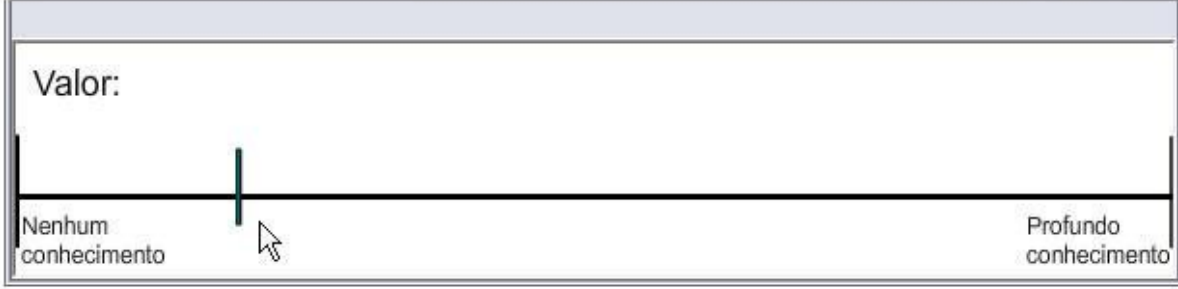

B

Valor: 437

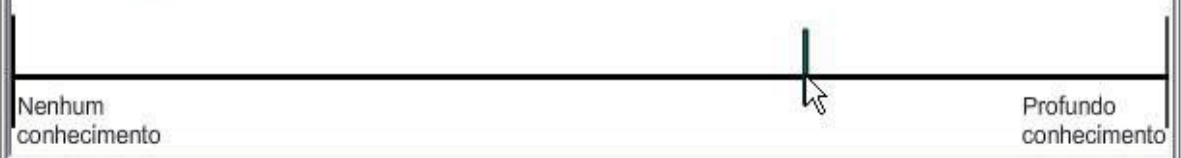

Figura 5. (A) Segmento de reta com uma pequena barra que se move conforme o mouse.

(B) Ao clicar, um valor é impresso na tela.

A quantificação da percepção foi realizada por meio de uma fórmula (1) para transformar os dados dos centímetros em escala de 10, onde $Q p$ é a quantificação da percepção; $v$ é o valor marcado pelo médico no segmento de reta e; $V$ é o valor total do segmento:

$$
\mathrm{Qp}=\mathrm{v} / \mathrm{V} \times 10
$$

\section{Resultados}

Após a implementação do objeto de aprendizagem, tem-se como resultados animações tridimensionais de alta qualidade demonstrando a variação de uma cabeça considerada perfeita a algumas com dismorfologias.

Na figura 6 é possível observar a sequência de imagens de uma animação que demonstra o crescimento da cabeça de um indivíduo portador da Plagiocefalia. Trata-se de uma craniossinostose, caracterizada por uma deformação no crânio causada pelo 
fechamento prematuro da sutura coronal unilateral. Nas figuras 6A e 6B pode-se observar a indicação da sutura que está relacionada a esta craniossinostose e uma breve descrição. Na figura 6C, é possível observar uma cabeça tridimensional considerada normal na visão superior e de perfil. Já na figura 6D, observam-se setas em vermelho que indicam onde ocorrerá a deformação no crânio. E finalmente, na figura 6E, visualiza-se uma cabeça com a dismorfologia.

Na figura 7 é possível observar a sequência de imagens de uma animação que demonstra um indivíduo portador da micrognatia. Trata-se de uma deformação na mandíbula caracterizada pelo queixo pequeno. Finalmente, a figura 8 mostra as telas de rotação da cabeça em 3D de um indivíduo portador da Escafocafalia. Esta é a deformação mais comum que ocorre no crânio, sendo causada pela fusão prematura da sutura sagital. O objeto de aprendizagem procurou implementar o máximo possível de interação do profissional com as imagens, visto que através das setas do teclado consegue-se visualizar a cabeça tridimensional em $360^{\circ}$.

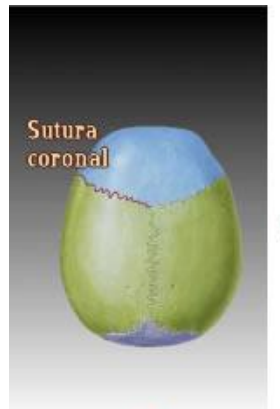

A

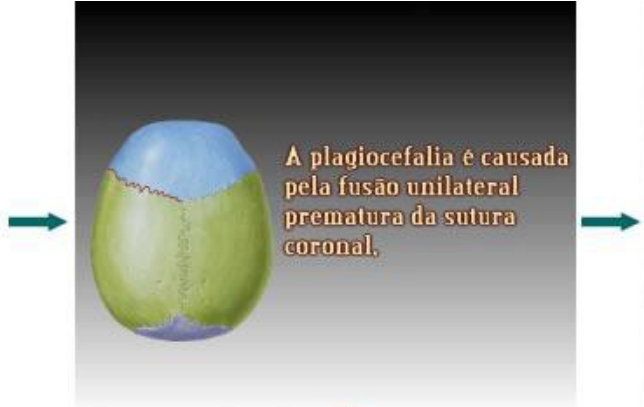

B

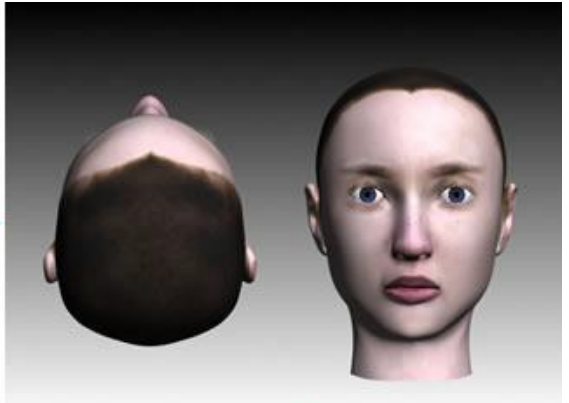

C

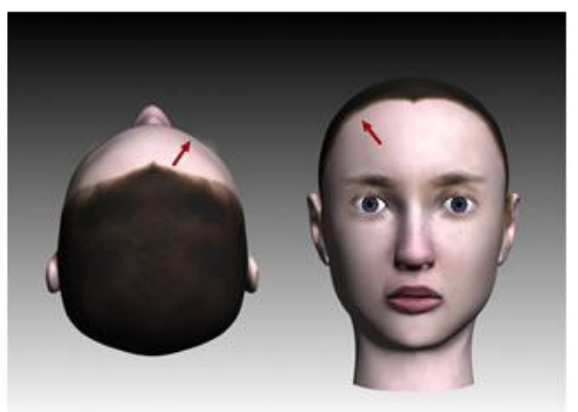

D

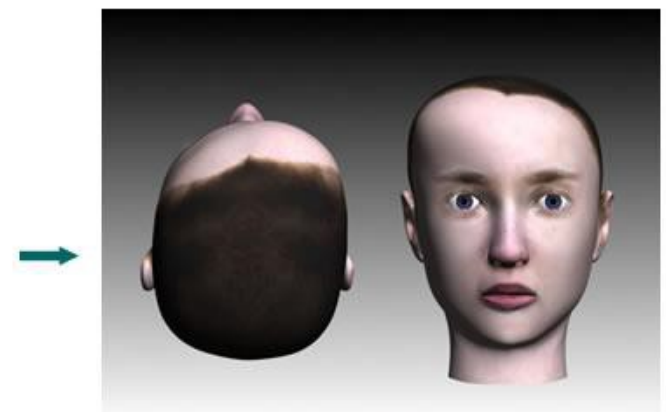

E

Figura 6. (A e B) Indicação da sutura. (C) Cabeça 3D considerada normal. (D) Indicação de onde ocorrerá a deformação. (E) Cabeça 3D final com a plagiocefalia.

Como resultado da avaliação do objeto de aprendizagem proposto obteve-se resposta de 27 pediatras sobre as questões de auto-percepção. No módulo de Craniossinostoses as médias e o desvio padrão antes e depois da visualização do objeto de aprendizagem foram de 2,62 $( \pm 2,14)$ e 6,44 $( \pm 1,96)$, respectivamente. A diferença entre as médias foi de 3,81 $( \pm 2,80)$, sendo $\mathrm{p}<0,05$. Posteriormente, no módulo de Micrognatia, a média e o desvio padrão antes da visualização do objeto de aprendizagem foi de $3,50( \pm 2,43)$ e depois foi de $6,67( \pm 1,90)$. A diferença entre as médias foi de 3,17 ( $\pm 2,59)$, sendo $\mathrm{p}<0,05$. Por fim, no módulo Macrognatia, as médias 
e o desvio padrão antes e depois da visualização do objeto de aprendizagem foram 3,06 $( \pm 2,32)$ e $6,71( \pm 1,92)$, respectivamente. A diferença entre as médias foi de 3,65 ( \pm 2,81 ), sendo $\mathrm{p}<0,05$.

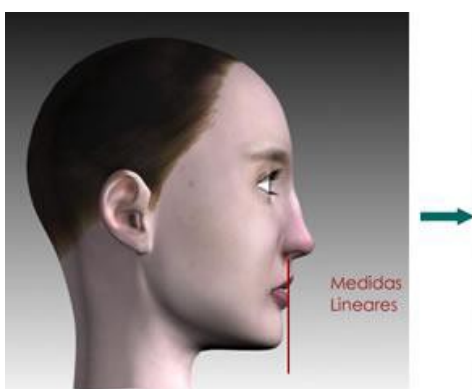

A

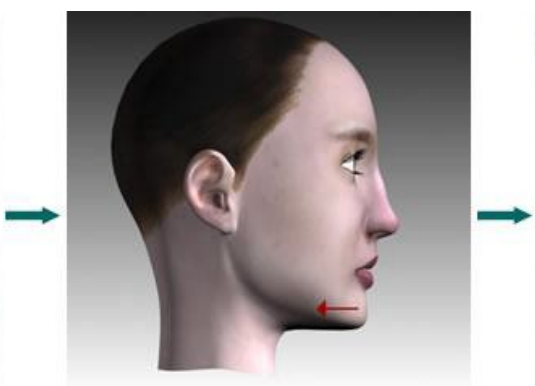

B

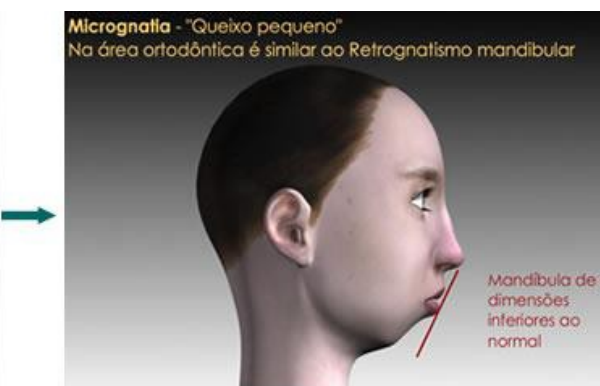

C

Figura 7. (A) Indicação das medidas lineares de uma mandíbula considerada normal. (B) Indicação de onde ocorrerá a deformação. (C) Cabeça 3D final com a micrognatia e informações adicionais sobre esta dismorfologia.

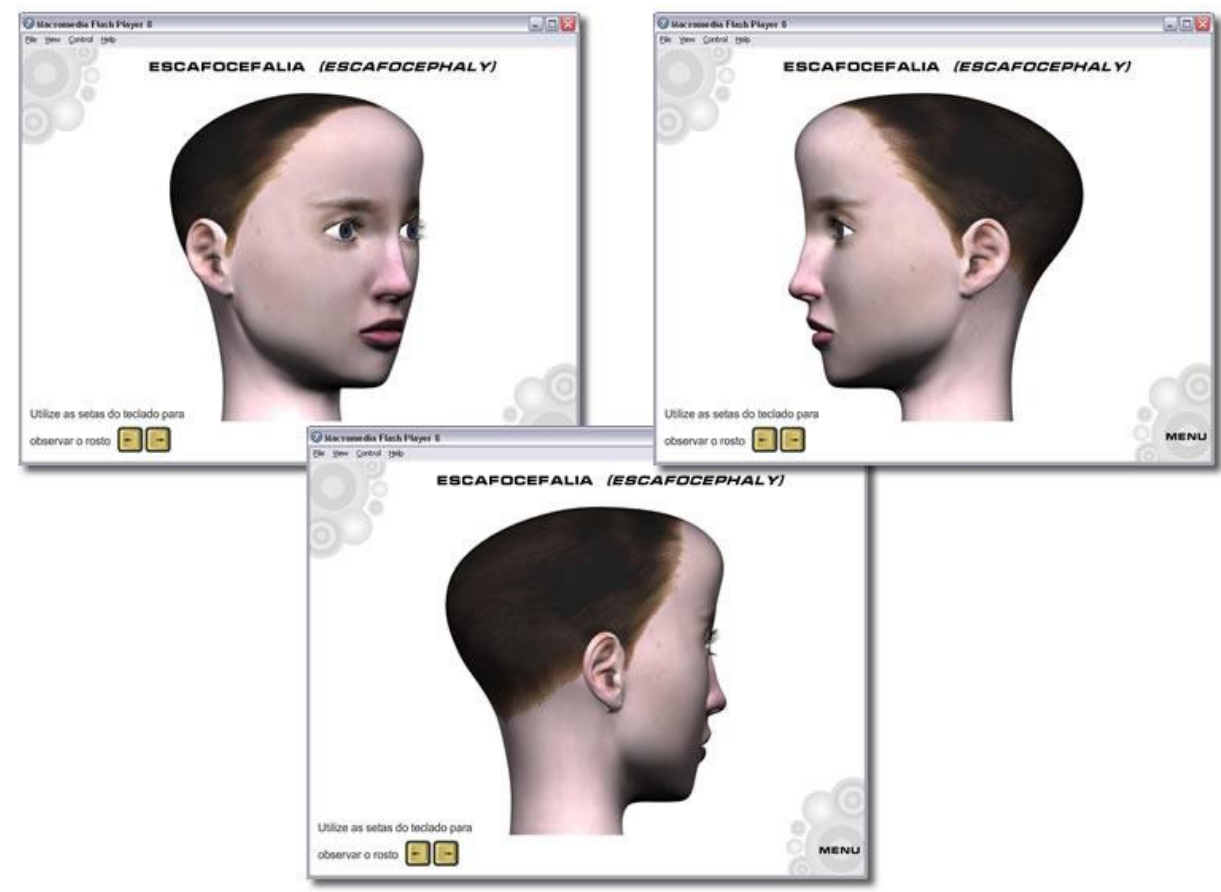

Figura 8. Telas com imagens da rotação da cabeça 3D (escafocefalia).

\section{Conclusões}

Pode-se dizer que modelos tridimensionais de morfologia facial são, atualmente, elementos de grande impacto na genética clínica quando se trata de estudos no desenvolvimento do crânio e da face (HAMMOND, 2007). Através da avaliação realizada neste estudo, percebe-se a importância que os objetos de aprendizagem em 3D podem ter na educação médica, incluindo o estudo da detecção das dismorfologias craniofaciais, visto que houve uma melhora estatisticamente significante das diferenças das médias sobre a compreensão antes e depois da utilização dos mesmos. 
Ademais, a identificação de indivíduos portadores de dismorfologias no estudo da genética não pode ter êxito sem a participação dos clínicos iniciais que compõem o atendimento primário a um paciente, ou seja, esses profissionais devem ser capazes de identificar pacientes afetados através da análise e detecção das dismorfologias. Desta forma, o objeto de aprendizagem é visto como um elemento motivador para a promoção do reconhecimento craniofacial de dismorfologias.

Com os dados apresentados neste estudo é possível iniciar uma reflexão sobre o grau de importância da utilização de modelos tridimensionais de morfologia facial no estudo do desenvolvimento das dismorfologias craniofaciais. A quantidade de deformações existentes é bastante extensa, entretanto, estima-se que propostas como essas sejam viáveis para que os profissionais de saúde, que não são especialistas na área da genética, tornem-se capazes de identificar dismorfologias, ao menos em um diagnóstico preliminar, com o auxílio destes recursos tecnológicos intuitivos, interativos e de fácil manuseio.

\section{Referências}

BESERRA, C. A.; TRINDADE, C. C.; SOUZA, P. R.; MAGALHÃES, C. V. C.; SANTOS, R. E. S. Aplicação de técnicas de aprendizagem de máquina em objetos de aprendizagem baseado em software: um mapeamento sistemático a partir das publicações do SBIE. Revista RENOTE, v. 12, n. 1, julho/2014.

BURITY, C. H. F.; CARDOZO, S. V. Atlas digital de morfologia comparada: uma ferramenta complementar ao ensino de histologia e histopatologia. Revista UNIABEU. v. 7, n. 16, 2014.

CANI, M. P. Towards Expressive 3D Modeling: new challenges for geometric computing. Proceedings. SOCG'14 Proceedings of the thirtieth annual Symposium on Computational Geometry. June 2014.

DEV, P. Tutorial: Imaging and Visualization in Medical Education. IEEE Computer Graphics and Applications, v. 19, n. 3, p. 21-23. 1999.

EL-KHALILI, N. and Brodlie, K. Architectural Design Issues for Web-Based Virtual Reality Training Systems. Proceedings... Proceedings of the International Conference on Web-based Modeling Simulation. Fishwick, P.; Hill, D.; Smith, R. (Eds), p. 153-158, San Diego, California, 1998.

FERREIRA, L. F. Usando objetos educacionais baseados em realidade virtual em ambientes de apoio a construção de conhecimento e aprendizagem de técnicas videocirúrgicas. Anais do II Ciclo de Palestras Novas Tecnologias na Educação. Porto Alegre, 2004.

GLUZ, J. C.; VICARI, R. M. MILOS: Infraestrutura de Agentes para Suporte a Objetos de Aprendizagem OBAA. Anais do XXI Simpósio Brasileiro de Informática na Educação. João Pessoa, 2010.

HAMMOND, P. The use of 3D face shape modelling in dysmorphology. Archives of Disease in Childhood, v. 92, n. 12, p. 1120-1126. 2007.

IEEE Learning Technology Standards Committee (LTSC). Draft Standard for Learning Object Metadata. Institute of Electrical and Electronics Engineers, Inc., 2000 . 
LIMA JÚNIOR, E.; KNOPFHOLZ, J.; MENINI, C. M. Estresse Durante Cursos de Suporte Avançado de Vida em Cardiologia. É Importante no Aprendizado de Habilidades. Arquivos Brasileiros de Cardiologia, v. 79, n. 6, p. 585-588. 2002.

GROSS, M. H. Computer graphics in medicine: from visualization to surgery simulation. SIGGRAPH Comput. Graph., v. 32, n. 1, p. 53-56. 1998.

MEDICINENET.COM. Definition of dysmorphology. Disponível em: http://www.medterms.com/script/main/art.asp?articlekey=3140. Acesso em: 01 jul. 2014.

SILVEIRA NETO, W. D.; KREPSKY, A. Técnicas de Animação em ambientes Tridimensionais. Revista Eletrônica de Sistemas de Informação, v. 4, n. 5.2005

SANTA-ROSA, J. G.; STRUCHINER, M. Tecnologia Educacional no Contexto do Ensino de Histologia: Pesquisa e Desenvolvimento de um Ambiente Virtual de Ensino e Aprendizagem. Revista Brasileira de Educação Médica, v. 35, n. 2. Rio de Janeiro, 2011.

SANTOS, L. M. A.; FLORES, M. L. P.; TAROUCO, L. M. R. Objeto de Aprendizagem: Teoria Instrutiva Apoiada por Computador. Anais do Ciclo de Palestras Novas Tecnologias na Educação, v. 6, n. 2, dezembro, 2007.

SEBASTIANI, R. L.; BEZ, M. R.; FLORES, C. D. Ensino de medicina apoiado pelo processamento de exames médicos baseados em imagem. Anais do XX Simpósio Brasileiro de Informática na Educação. Florianópolis, 2009.

SILVA, R. W. A. D. Educação A Distância em Ambientes de Aprendizagem Matemática Auxiliada pela Realidade Virtual. (Dissertação de Mestrado). Programa de Pós-Graduação em Engenharia de Produção, Universidade Federal de Santa Catarina, Florianópolis, 2001.

TORI, A. A.; LEITE, K. T. M.; PACOLLA, S. A. O.; AMANTINI, S. R.; MACHADO, M. A. A. M. Modelagem realística de cabeça infantil para aplicação em simulador de anestesia odontológica. Revista SENAC de Iniciação Científica, Tecnológica e Artística, v. 4, n. 3, 2014.

TORI, Romero; KIRNER, Claudio. Fundamentos de Realidade Virtual. In: Fundamentos e Tecnologia de realidade Virtual e Aumentada. Symposium on Virtual Reality, Belém, 2006.

VILAR, G.; D. S. VILAR. Processos Colaborativos e Tecnologias da Informação Aplicados ao Ensino de Medicina. Colabor@, v. 2, n. 7. 2004.

WILEY, D. A. Learning object design and sequencing theory. Doctoral dissertation, Brigham Young University, 2000.

\footnotetext{
i 3DS Max é marca registrada da Autodesk, Inc.

ii MOODLE é o acrônimo de "Modular Object-Oriented Dynamic Learning Environment" e está disponível em www.moodle.org.
} 